\title{
Exciton storage in type-Il quantum dots using the optical Aharonov-Bohm effect
}

\author{
Juan I. Climente and Josep Planelles ${ }^{a}$ \\ Departament de Química Física i Analítica, Universitat Jaume I, E-12080 Castelló, Spain
}

(Received 11 April 2014; accepted 30 April 2014; published online 12 May 2014)

\begin{abstract}
We investigate the bright-to-dark exciton conversion efficiency in type-II quantum dots subject to a perpendicular magnetic field. To this end, we take the exciton storage protocol recently proposed by Simonin and co-workers [Phys. Rev. B 89, 075304 (2014)] and simulate its coherent dynamics. We confirm the storage is efficient in perfectly circular structures subject to weak external electric fields, where adiabatic evolution is dominant. In practice, however, the efficiency rapidly degrades with symmetry lowering. Besides, the use of excited states is likely unfeasible owing to the fast decay rates. We then propose an adaptation of the protocol which does not suffer from these limitations. (C) 2014 AIP Publishing LLC. [http://dx.doi.org/10.1063/1.4876479]
\end{abstract}

Exciton storage in semiconductor nanostructures is a long-pursued goal in condensed matter physics. Potential applications range from optical ${ }^{1}$ and quantum ${ }^{2}$ memory elements to light retarders for optical communication and smart pixels,${ }^{3}$ allowing image detection, processing, and generation in one semiconductor element. ${ }^{4}$

To date, most techniques for exciton storage rely on strong electric fields which dissociate the exciton confined in the nanostructure. This was, e.g., the case for excitons in quantum wells, ${ }^{1,5}$ natural quantum dots (QDs), ${ }^{6}$ quantum dot molecules, ${ }^{3}$ quantum posts ${ }^{7}$ and rods, ${ }^{8}$ or quantum rings. ${ }^{9}$ Recently, however, Simonin and co-workers studied the behavior of excitons in semiconductor quantum rings subject to perpendicular magnetic and in-plane electric fields. ${ }^{10}$ Based on their stationary results, they envisaged a protocol for exciton storage through a sequence of magnetic flux and electric field modulations. The underlying idea is that the initially bright exciton (envelope angular momentum $L=0$ ) is transformed into a dark one $(L=1)$ by means of the AharonovBohm (AB) optical effect. ${ }^{11}$ As opposed to previous protocols, here electron-hole recombination is prevented by angular momentum selection rules. The exciton is then expected to remain bound and no strong electric fields are needed.

In this work, we extend the study of the exciton storage mechanism proposed in Ref. 10 by simulating the corresponding dynamics. This allows us to assess on the feasibility of the adiabatic or diabatic steps required by the protocol. The exciton storage protocol conceived in Ref. 10 was originally proposed for InGaAs quantum rings with slightly polarized electron and holes, where the optical $A B$ effect should be observable. For convenience, however, we propose using type-II ZnTe/ZnSe QDs instead. ${ }^{12}$ Stacks of such QDs have been fabricated and studied by Sellers and co-workers. ${ }^{13}$ They found that the hole in these structures is localized inside the dot, while the electron is outside, orbiting radially around the dot, bound by Coulomb attraction. This is illustrated schematically in Fig. 1(a), which shows side and top views of the system. We note that type-II QDs present two clear advantages with respect to InGaAs rings. First, the difference between electron and hole radii, $\left|R_{e}-R_{h}\right|$, is much larger. As a result, so is the magnetic flux trapped between the two carriers. This implies that weaker magnetic fields are required to induce $\mathrm{AB}$ oscillations. Second, the radii are smaller. This gives rise to larger energy gaps, so that cryogenic temperatures are no longer required to observe the optical $\mathrm{AB}$ effect. ${ }^{13}$

The Hamiltonian describing the exciton is given by

$$
H=H_{e}+H_{h}+U_{e h},
$$

where $H_{e}$ is the single-particle electron Hamiltonian

$$
H_{e}=\frac{\hbar^{2}}{2 m_{e} R_{e}^{2}}\left(-i \frac{\partial}{\partial \theta_{e}}+\frac{\phi_{e}}{\phi_{0}}\right)^{2}-e F R_{e} \cos \theta_{e},
$$

and $H_{h}$ is that of the hole

$$
H_{h}=\frac{\hbar^{2}}{2 m_{h} R_{h}^{2}}\left(i \frac{\partial}{\partial \theta_{h}}+\frac{\phi_{h}}{\phi_{0}}\right)^{2}+e F R_{h} \cos \theta_{h} .
$$

Here, $m_{j}$ is the effective mass of the carrier $j(j=e, h), R_{j}$ the radius of its orbit, $\theta_{j}$ its angular coordinate, $\phi_{j}=\pi R_{j}^{2} B$ the magnetic flux induced by a magnetic field $B, \phi_{0}=h c / e$ the flux quantum, $e$ the electron charge, and $F$ the in-plane electric field. In turn, $U_{e h}$ is the electron-hole Coulomb interaction

$$
U_{e h}=-\frac{e^{2}}{\epsilon \sqrt{R_{e}^{2}+R_{h}^{2}}} \frac{1}{\sqrt{1-r \cos \left(\theta_{e}-\theta_{h}\right)}},
$$

with $\epsilon$ the dielectric constant and $r=2 R_{e} R_{h} /\left(R_{e}^{2}+R_{h}^{2}\right)$.

Hamiltonian (1) is solved numerically using finite differences. Its corresponding time-dependent Schrödinger equation, projected on the numerical eigenstates, can be written as

$$
i \hbar \frac{d}{d t} \mathbb{C}=\mathbb{H} \mathbb{C}
$$

which is equivalent to 


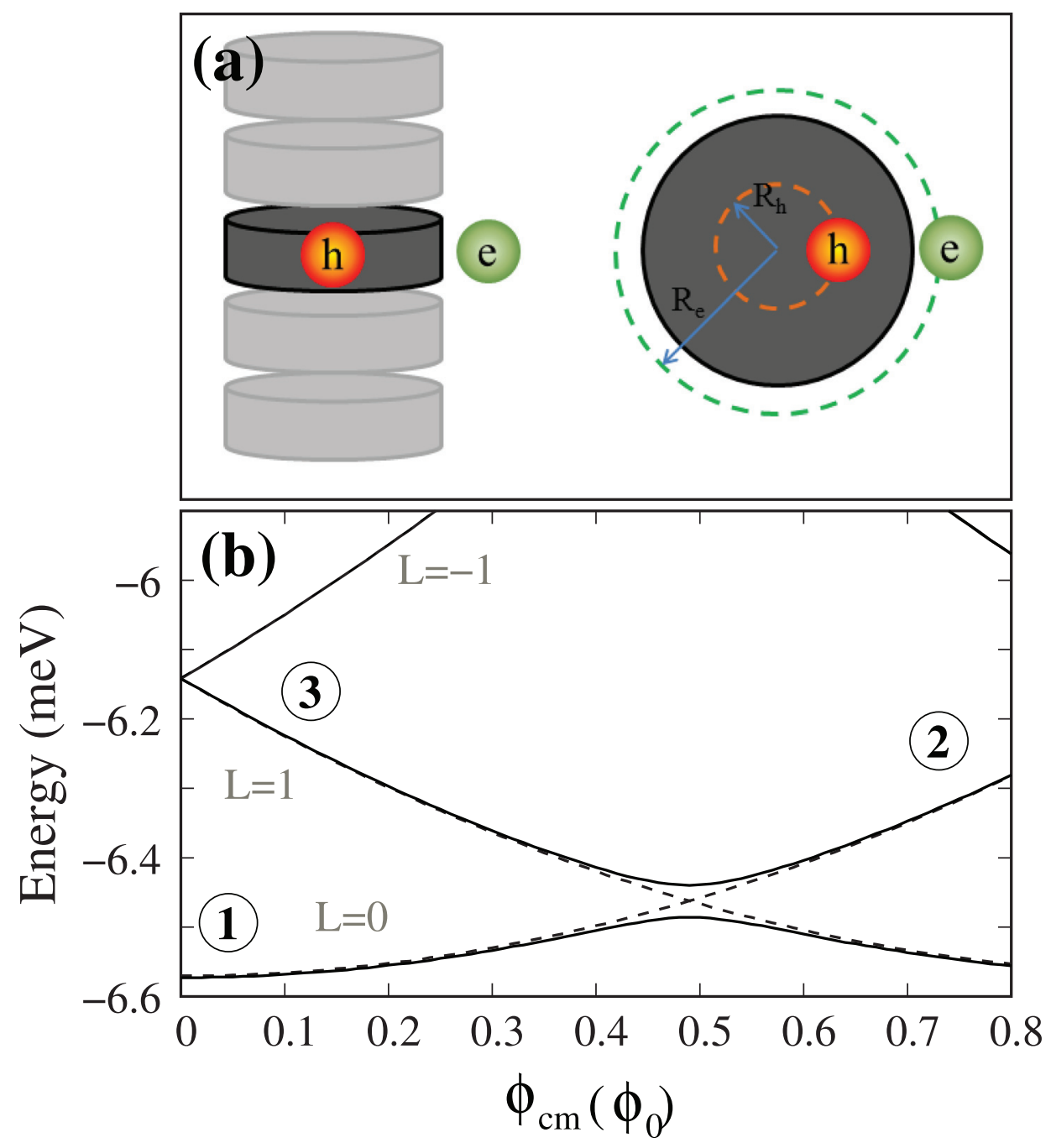

FIG. 1. (a) Side (left) and top (right) view of the type-II QD system we study. (b) Exciton energy spectrum as a function of the center of mass magnetic flux. Dashed lines: $F=0 \mathrm{kV} / \mathrm{cm}$. Solid lines: $F=20 \mathrm{~V} / \mathrm{cm}$. The encircled numbers indicate the steps of the storage mechanism.

$$
\mathbb{C}(t)=\mathbb{U}(t) \mathbb{C}(0),
$$

where $\mathbb{C}(0)$ is the vector of stationary eigenstates at $t=0$. Since both the external fields $F$ and $\phi$ vary in time, the Hamiltonian $H$ is time-dependent itself. The time evolution operator $\mathbb{U}(t)$ is then given by the Trotter approximation

$$
\mathbb{U}(t)=\lim _{n \rightarrow \infty} \prod_{j=0}^{n-1} \mathbb{U}_{j},
$$

with $\mathbb{U}_{j}=e^{-i \mathbb{H}_{j} t_{j} / \hbar}$, where $\mathbb{H}_{j}$ is the Hamiltonian projection at time $t_{j}=t / n * j$. In practice, $n$ in Eq. (7) is taken large enough to ensure a correct chronological order of the infinitesimal time evolution operators.

Figure 1(b) shows the exciton energy spectrum as a function of the angular "center of mass" magnetic flux, $\phi_{c m}=\pi B\left(R_{e}^{2}-R_{h}^{2}\right)$. Dashed lines are used for the case without external electric field. One can see that the ground state changes at $\phi_{c m}=0.5 \phi_{0}$ from $L=0$ (bright exciton) to $L=1$ (dark exciton). When the electric field is switched on (solid lines), the level crossings are replaced by anticrossings. The encircled numbers in the figure allow us to follow the exciton storage protocol proposed in Ref. 10. The protocol starts with the optically generated bright exciton at (1). The magnetic flux is then increased beyond $\phi_{c m}=0.5 \phi_{0}$. In the absence of electric fields, there is perfect circular symmetry, so $L=0$ and
$L=1$ states are orthogonal and the system is expected to evolve adiabatically until (2). At this point, the magnetic flux is lowered back to zero but in the presence of an external electric field. This lifts angular momentum symmetry and opens the anticrossing gap between the former $L=0$ and $L=1 \mathrm{lev}$ els. As a result, the adiabatic evolution now leads to (3), which is a dark state with long radiative lifetime. The bright exciton can be retrieved following the inverse steps.

Figure 2 shows the dynamics corresponding to the protocol described above. Panel (a) shows the sequence of external fields as a function of time, while panel (b) shows the weight of the bright exciton. The time-dependent wave function $\Psi(t)$ can be expressed as an expansion of the stationary eigenfunctions of Hamiltonian (1) at time zero, $\varphi_{L}$ :

$$
\Psi(t)=\sum_{L} c_{L}(t) \varphi_{L} e^{-i E_{L} t / \hbar}
$$

where $E_{L}$ is the energy of the state with azimuthal angular momentum $L$-at time zero angular symmetry is preserved. Then, the weight of the bright exciton at any time is given by $\left|c_{L=0}(t)\right|^{2}$.

We consider three electric field values. For $F=0 \mathrm{~V} / \mathrm{cm}$ (red line in Fig. 2(b)), the exciton remains bright all the time. In other words, it moves from (1) to (2) and back again. Switching on an electric field as small as $F=5 \mathrm{~V} / \mathrm{cm}$ (blue 


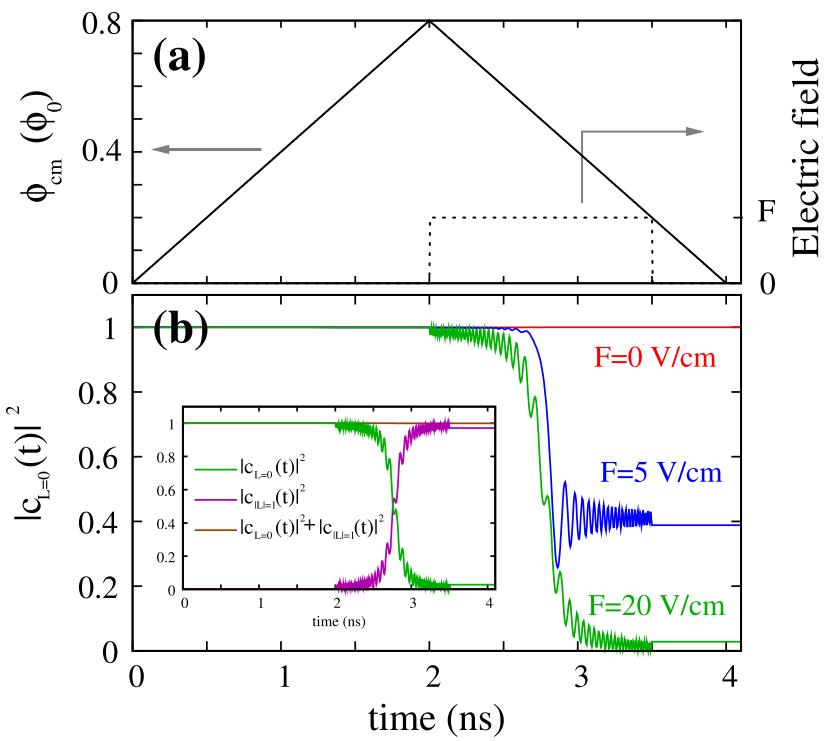

FIG. 2. (a) Sequence of magnetic flux (solid line) and electric field (dashed line) applied in the storage mechanism proposed in Ref. 10. (b) Time evolution of the bright exciton component. The inset in (b) compares the weight of $L=0$ and $L=1$ exciton components.

line) already has a strong influence on the time evolution. The weight of the bright exciton component decreases and oscillates around 0.4 . Using $F=20 \mathrm{~V} / \mathrm{cm}$ instead (green line), the weight of the bright exciton becomes really small, oscillating around 0.02 . This confirms that the adiabatic transition from (2) to (3) (can be very efficient, thus supporting the bright-to-dark exciton conversion proposed in Ref. 10.

The inset in Fig. 2(b) shows the time evolution of the $\mathrm{L}=0$ and $|\mathrm{L}|=1$ exciton components at $\mathrm{F}=20 \mathrm{~V} / \mathrm{cm}$. As can be seen, the exciton composition oscillates exclusively between these low-energy states. This is generally the case for weak electric fields. The reason is that, at first order, the electric field couples only states differing in $\Delta L= \pm 1$.

One can check that the asymptotic probability of the transitions we calculate numerically is consistent with the simple Landau-Zener formula for two anticrossing states, ${ }^{14,15}$ when the electric field is of up to $F \approx 10 \mathrm{~V} / \mathrm{cm}$. However, the Landau-Zener theory starts failing for $F=20 \mathrm{~V} / \mathrm{cm}$, as higher perturbations start becoming important. Indeed, contrary to what one might expect from Fig. 2 and the Landau-Zener formula, further increasing the electric field does not improve the efficiency of the exciton storage mechanism. Fig. 3 compares the time evolution for a weak $(F=20 \mathrm{~V} / \mathrm{cm}$, green line) and a moderately large field $(F=100 \mathrm{~V} / \mathrm{cm}$, pink line). With increasing field, the amplitude of the oscillations becomes wider, so that the final value of $c_{L=0}$ is strongly dependent on the time at which the field is switched off. Besides, the bright-to-dark conversion efficiency gets worse (about 0.2 in the figure). The lower conversion efficiency is a consequence of the strong field enabling diabatic evolution from (2) to (1), instead of the desired adiabatic evolution to (3). In turn, the wider oscillations can be understood from the fact that, starting from a $\mathrm{L}=0$ exciton, the weight of the other components is given at first order perturbation by

$$
\left|c_{L}\right|^{2}=V_{0 L}^{2} \frac{\sin ^{2} \omega_{0 L} t}{\omega_{0 L}^{2}}
$$

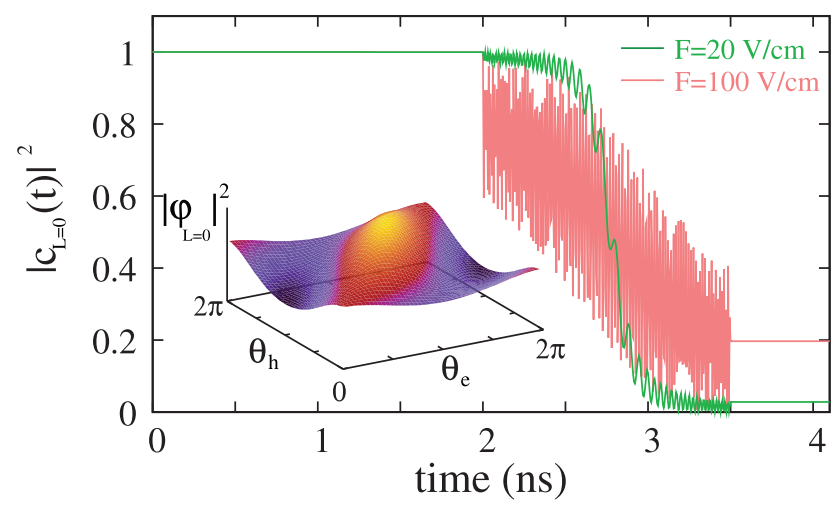

FIG. 3. Same as Fig. 2(b) but now comparing weak and moderately strong electric fields. The inset shows the $L=0$ exciton charge density at $F=20 \mathrm{~V} / \mathrm{cm}$.

where $V_{0 L}=\left\langle\varphi_{0}|V| \varphi_{L}\right\rangle$ is the electric field matrix element, with $V=e F\left(R_{e} \cos \theta_{e}-R_{h} \cos \theta_{h}\right)$, and $\omega_{0 L}=\left(E_{L}-E_{0}\right) / \hbar$ is the oscillation frequency. It is clear from Eq. (9) that the amplitude of the oscillations is proportional to the field $F$.

It is worth noting that the weak electric fields required by the protocol allow one to obtain excitons which are dark inspite of the electron and hole not being dissociated. This is confirmed in the inset in Fig. 3, which shows the exciton ground state at $F=20 \mathrm{~V} / \mathrm{cm}$. The probability of finding the electron and hole together is high for any angle, which is the signature of quasi-free excitons. ${ }^{10}$

So far, we have considered that in the absence of electric fields there is perfect circular symmetry, so that $L=0$ and $L=1$ states cross each other. In practice, both quantum rings and type-II QDs are expected to present deviations from this ideal limit. Ellipticity is generally the most important one, but it preserves $C_{2}$ symmetry, so that the two first states still cross. ${ }^{16-18}$ Further lowering of the symmetry would however give rise to anticrossing gaps between the two states. ${ }^{18,19}$ Since the results in Fig. 2 reveal very high sensitivity to electric fields, one suspects that even a small anticrossing gap may play an important role in the transition from (1) to (2). In order to test this, in Fig. 4, we consider the exciton storage
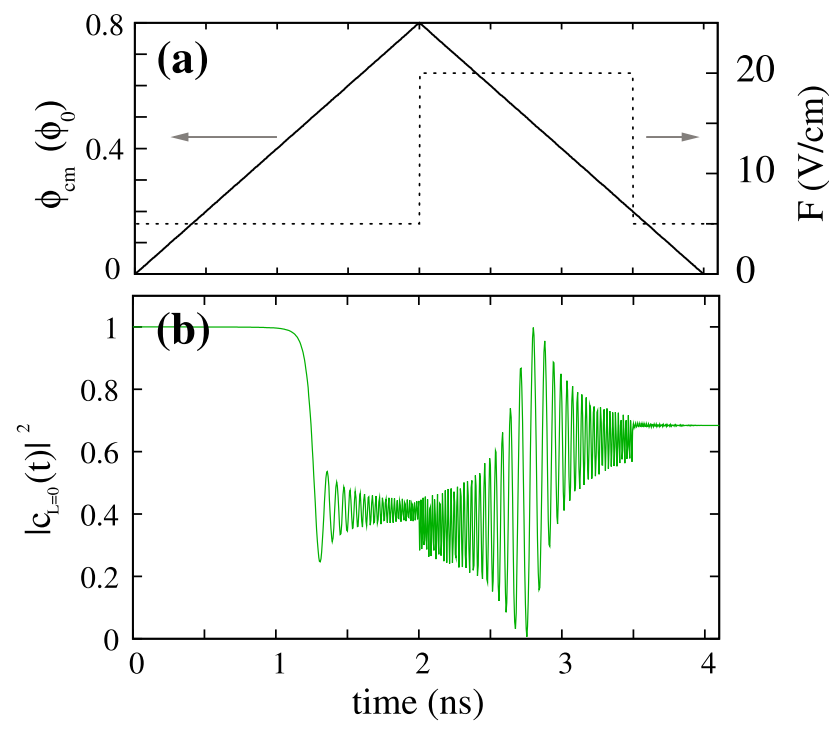

FIG. 4. Same as Fig. 2 (with $F=20 \mathrm{~V} / \mathrm{cm}$ ) but in the presence of a background electric field $F_{0}=5 \mathrm{~V} / \mathrm{cm}$ accounting for deviations from circular symmetry. 

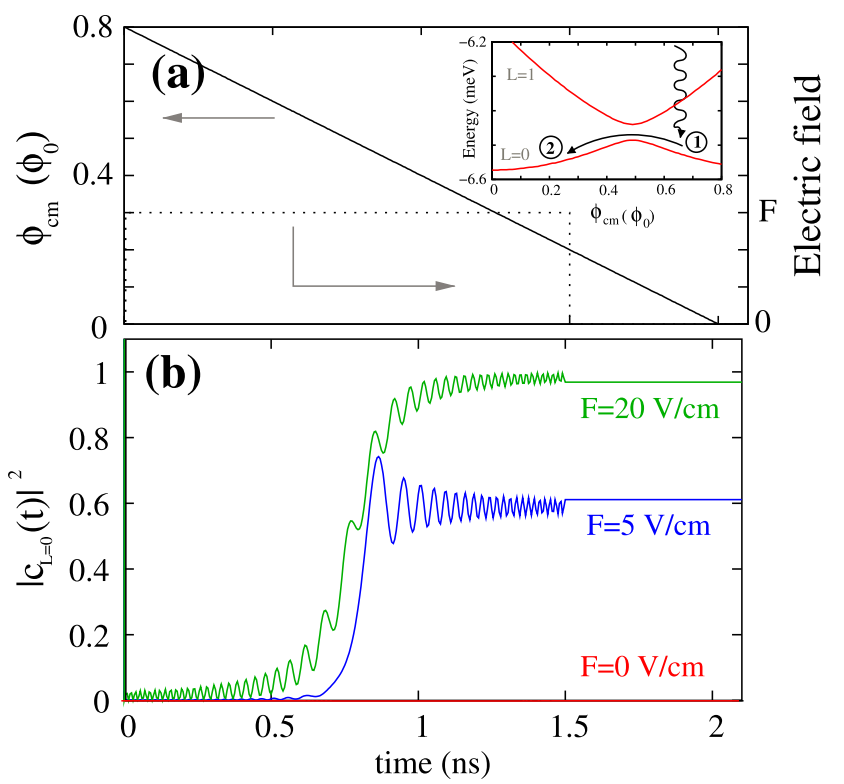

FIG. 5. Same as Fig. 2 but for the exciton storage and conversion protocol proposed in this work. The inset in (a) shows the exciton energy spectrum, with arrows denoting the steps of the protocol.

protocol of Ref. 10 but assuming a weak background electric field of $F_{0}=5 \mathrm{~V} / \mathrm{cm}$, which accounts for some minor anisotropy removing rotational symmetry. This gives rise to a gap of $\sim 0.04 \mathrm{meV}$ between $L=0$ and $L=1$ states. Despite the small magnitude of the anticrossing, one can see that the exciton evolution is severely affected, the dynamics being more complicated and less efficient than in the absence of background field (cf. green line in Fig. 2).

The need of perfect rotational symmetry poses an important practical challenge for the implementation of the exciton storage protocol proposed in Ref. 10, but further challenges ensue from the competition with other dynamical processes. Namely, (i) the transition from (1) to (2) must be operated in a timescale faster than —or at least comparable to - the bright exciton radiative decay rate; (ii) the excited states, such as (2) and (3) tend to decay non-radiatively to the ground state even at low temperatures through acoustic phonon emission. In both cases, the decay rates are of the order of ns. ${ }^{20,21}$ It is unlikely that strong magnetic fields can be controlled on such timescales, and in any case the stored dark excitonbeing excited-will have too short a lifetime.

To overcome the abovementioned handicaps, in what follows we propose a variant of the exciton storage protocol of Simonin and co-workers. The idea is summarized in the inset of Fig. 5(a). The QD is initially set above $\phi_{c m}=0.5 \phi_{0}$. Next, it is populated by means of non-resonant optical excitation. The exciton decays quickly from the continuum into the ground state (ps). Because of the optical $\mathrm{AB}$ effect the ground state is a dark exciton, point (1) in the inset, with long radiative lifetime. When one wants to convert the dark exciton into a bright one, an electric field is switched on and the magnetic flux decreased below $\phi_{c m}=0.5 \phi_{0}$. This enables the adiabatic transition from (1) to (2) (see inset).

The sequence of external fields corresponding to this protocol is plotted in Fig. 5(a), and the dynamics in Fig. 5(b). As seen in the figure, the initially dark exciton $\left(\left|c_{L=0}\right|^{2}=0\right)$ is efficiently converted into a bright one using weak electric fields $\left(\left|c_{L=0}\right|^{2}=0.98\right.$ for $\left.F=20 \mathrm{~V} / \mathrm{cm}\right)$. The underlying physics is the same as in the previous protocol (optical $\mathrm{AB}$ effect, coherent oscillations between $L=0$ and $L=1$ excitons), but several practical advantages are present: (i) the need of zero-gap crossings is removed, (ii) competition with non-radiative processes is excluded because only the ground state is used, and (iii) the dark exciton is formed without relying on the bright exciton recombination times. As a matter of fact, in Fig. 5, we use ns timescale for convenience of the presentation (the period of oscillations is fraction of ns), but in practice one could afford much longer manipulation times, thus making magnetic control feasible. ${ }^{22}$

In conclusion, we have proposed a protocol for exciton storage and revival in type-II QDs. The protocol is built on that recently outlined by Simonin and co-workers, ${ }^{10}$ which relies on the use of the optical Aharonov-Bohm effect rather than strong electric fields. Our simulation of the exciton dynamics reveal that Simonin's protocol is indeed feasible as long as the system has rotational symmetry and the external field is weak enough for Landau-Zener tunneling to hold. By contrast, our variant is more robust against exciton relaxation and symmetry lowering.

Support from MINECO Project CTQ2011-27324 and UJI-Bancaixa P1-1B2011-01 is acknowledged.

${ }^{1}$ S. Zimmermann, A. Wixforth, J. P. Kotthaus, W. Wegscheider, and M. Bichler, Science 283, 1292 (1999).

${ }^{2}$ A. Boyer de la Giroday, N. Sköld, R. M. Stevenson, I. Farrer, D. A. Ritchie, and A. J. Shields, Phys. Rev. Lett. 106, 216802 (2011).

${ }^{3}$ T. Lundstrom, W. Schoenfeld, H. Lee, and P. M. Petroff, Science 286, 2312 (1999).

${ }^{4}$ J. P. Kotthaus, Science 286, 2286 (1999).

${ }^{5}$ A. G. Winbow, A. T. Hammack, L. V. Butov, and A. C. Gossard, Nano Lett. 7, 1349 (2007).

${ }^{6}$ A. A. High, A. T. Hammack, L. V. Butov, L. Mouchliadis, A. L. Ivanov, M. Hanson, and A. C. Gossard, Nano Lett. 9, 2094 (2009).

${ }^{7}$ H. J. Krenner, C. E. Pryor, J. He, and P. M. Petroff, Nano Lett. 8, 1750 (2008).

${ }^{8}$ R. M. Kraus, P. G. Lagoudakis, A. L. Rogach, D. V. Talapin, H. Weller, J. M. Lupton, and J. Feldmann, Phys. Rev. Lett. 98, 017401 (2007).

${ }^{9}$ A. M. Fischer, V. L. Campo, Jr., M. E. Portnoi, and R. A. Römer, Phys. Rev. Lett. 102, 096405 (2009).

${ }^{10}$ J. Simonin, C. R. Proetto, M. Pacheco, and Z. Barticevic, Phys. Rev. B 89, 075304 (2014).

${ }^{11}$ A. O. Govorov, S. E. Ulloa, K. Karrai, and R. J. Warburton, Phys. Rev. B 66, 081309 (2002).

${ }^{12}$ Input parameters: $m_{e}=0.16 m_{0}, m_{h}=0.60 m_{0}\left(m_{0}\right.$ is the free electron mass), $\epsilon=9.5, R_{e}=23.5 \mathrm{~nm}$, and $R_{h}=3.5 \mathrm{~nm}$.

${ }^{13}$ I. R. Sellers, V. R. Whiteside, I. L. Kuskovsky, A. O. Govorov, and B. D. McCombe, Phys. Rev. Lett. 100, 136405 (2008).

${ }^{14}$ A. C. Vutha, Eur. J. Phys. 31, 389 (2010).

${ }^{15}$ A. V. Shytov, Phys. Rev. A 70, 052708 (2004).

${ }^{16}$ J. Simonin, C. R. Proetto, Z. Barticevic, and G. Fuster, Phys. Rev. B 70, 205305 (2004).

${ }^{17}$ A. Bruno-Alfonso and A. Latgé, Phys. Rev. B 71, 125312 (2005).

${ }^{18}$ J. Planelles, F. Rajadell, and J. I. Climente, Nanotechnology 18, 375402 (2007).

${ }^{19}$ L. G. G. V. Dias da Silva, S. E. Ulloa, and A. O. Govorov, Phys. Rev. B 70, 155318 (2004).

${ }^{20}$ L. Jacak, P. Hawrylak, and A. Wojs, Quantum Dots (Springer Verlag, Berlin, 1998).

${ }^{21}$ M. Sugawara, Self-Assembled InGaAs/GaAs Quantum Dots (Academic Press, San Diego, 1999), Chap. 5.

${ }^{22} \mathrm{~A}$ limiting timescale will be set by the dark exciton radiative recombination rate. This process is forbidden by angular momentum conservation in circular QDs, but deviations from the symmetry will gradually lower the restriction. 